\title{
A deviation transfer vector loop construction method based on assembly constraint
}

\author{
Haitao Liu' ${ }^{1, ~ a ~ a n d ~ J i a n j u n ~ T a n g ~}{ }^{1, b^{*}}$ \\ ${ }^{1}$ AVIC Chengdu Aircraft Industrial (Group) Co., Ltd., Chengdu 610092, China \\ alhtbaby1261@163.com, btang8848@126.com
}

Keywords: assembly deviation transfer; precision prediction; vector loop; assembly constraint

Abstract. Effective build assembly deviation transfer path is the basis for fast and reliable assembly precision prediction, for which proposed a deviation transfer vector loop construction method based on assembly constraint. First datum reference frames, reference path, assembly connection point and other parameters were defined. Secondly the vector path of deviation transfer path between the two parts which had assembly constraints was established; Finally, according to assembly sequence, the deviation transfer path vector end to end, forming a assembly vector loop, and as a basis for constructing vector equation. A inner wing flap rocker was taken as the research example to establish the complete assembly deviation transfer vector loop and vector equation.

\section{Introduction}

In high precision manufacturing process of aircraft, precision equipment, etc., the assembly precision is one of the important factors that affect product performance. Due to the different assembly constraints and the parts dimensions, each part dimensions variation has different degree of influence on the whole assembly precision [1]. Better achieve prediction and control of assembly precision, the impact of components and assembly constraints for assembly precision need to be fully considered.

In the process of product assembly, deviation transfer path reflects the formation rule of the final product's precision, and it can also describe the impact on the product's precision of parts. Literature [2-4] proposed the concept of deviation vector model, but did not consider the deviation sensitivity on deviation cumulative in multidimensional environment. LIU [5] constructed a three-dimensional state space model of deviation transfer, but primarily made positioning element deviation as deviation source. Mathieu Mansuy [6] proposed a multidimensional vector direction of geometric elements segment, but did not consider the role of the assembly benchmark to deviation transfer.

For a comprehensive analysis of the impact parts geometrical elements on the assembly precision in the multidimensional space, to achieve a more reliable prediction of assembly precision, a deviation transfer vector loop construction method based on assembly constraints is proposed. To lay the foundation for accurate and efficient assembly precision prediction and control, deviation transfer path vector loop is constructed by defining assembly constraints, benchmark reference structural of components and deviation transfer path.

\section{Deviation transfer path based on assembly constraint}

Assembly parts are located through the locating datum in the process of product assembly. In the process of forming deviation transfer path, part's locating datum is called DRF (Datum Reference Frame). DP (Datum Path) in Fig. 1(2) is a path from the joint to the DRF which is connected with nominal dimension vectors.

Fig. 1 (1) descript design model consisting of the dock and the slider, $a_{1}, a_{2}, \ldots, a_{5}, U_{1}, \ldots U_{3}$ indicate the product design size. The $D P_{11}, D P_{12}, D P_{21}$ and $D P_{22}$ in Fig. 1 (2) are through the joint A, B and DRF, and along the vector of design size in Fig. 1 (1). 

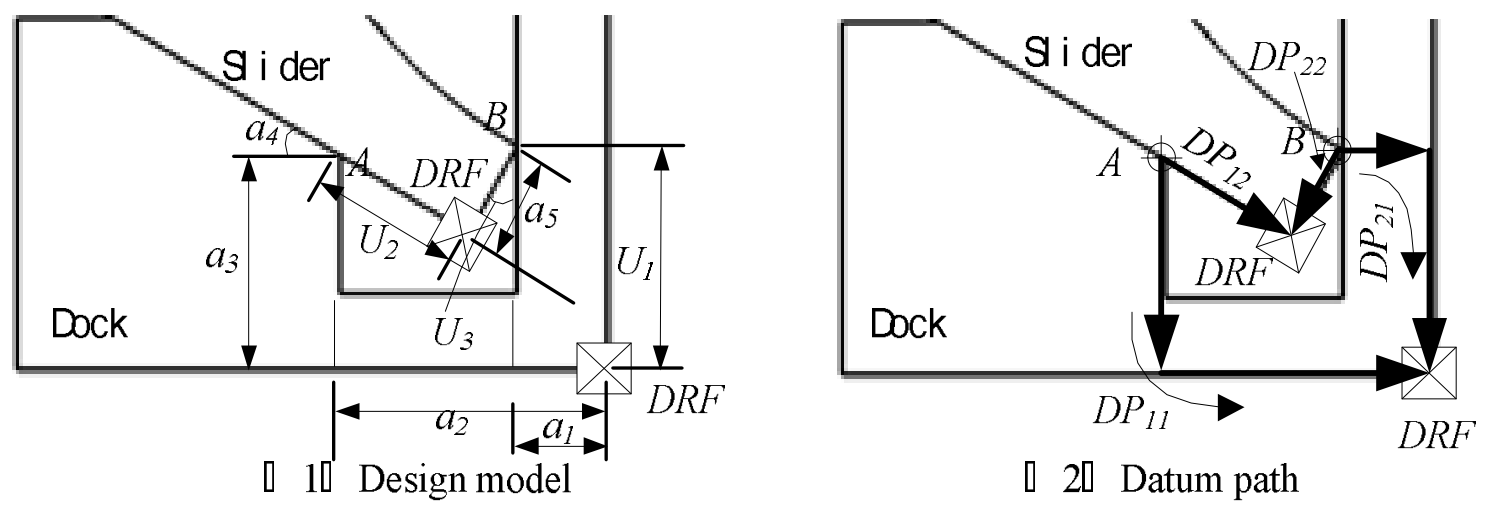

FIGURE 1: Deviation transfer datum path

The deviation transfer path of two constrained parts is along a DP, and through a joint. It must obey certain modeling rules as it passes through a part. It must:

1) Enter a part through a joint;

2) Follow the DP to the DRF in the part;

3) Follow a second DP leading to another joint;

4) Exit to the next adjacent part from the joint in the assembly.

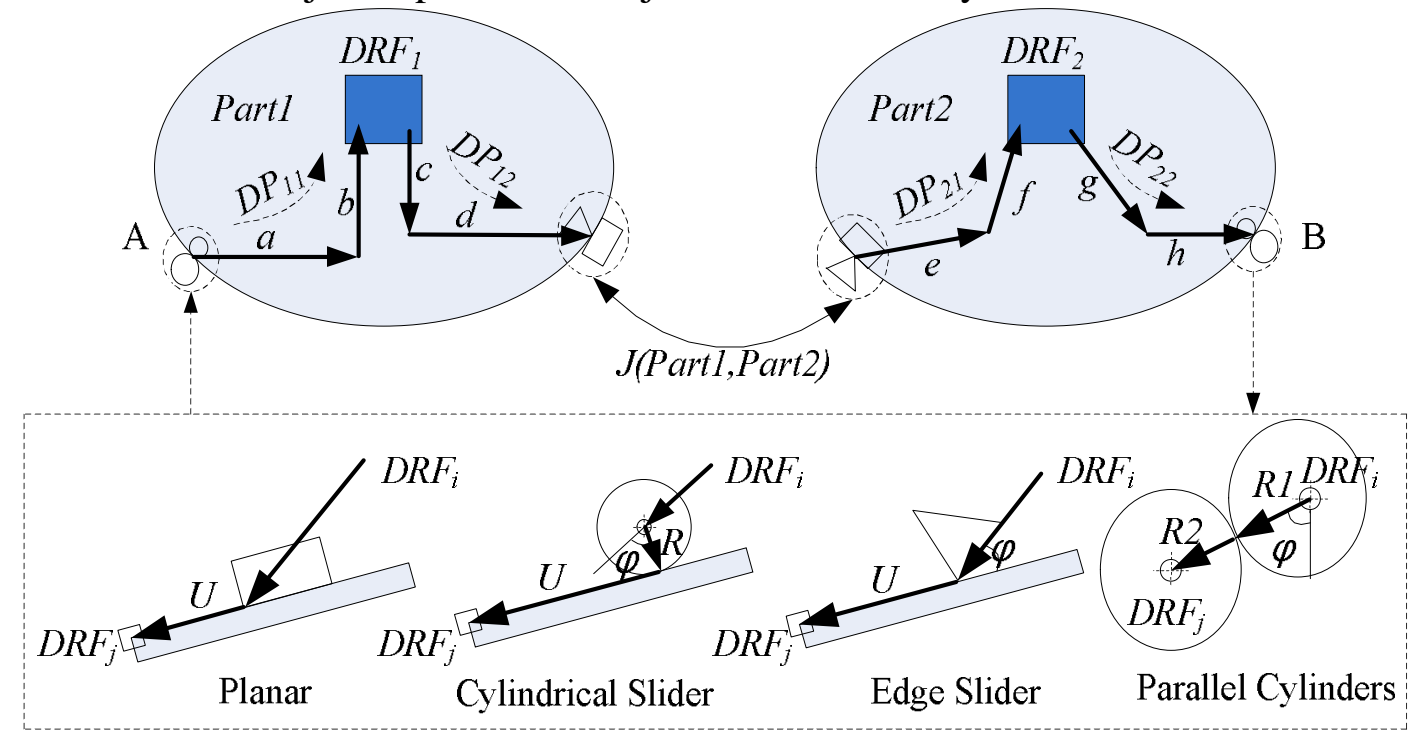

FIGURE 2: Deviation transfer path constructed by different kind of assembly constraints

The deviation transfer path of Part1 and Part2 is illustrated in Figure 2, which is formed by four DPs in Part1 and Part2 $\left(D P_{11}, D P_{12}, D P_{21}\right.$ and $\left.D P_{22}\right)$. They are created by dimension vectors $(a, b, c, d, e$, $f$, $g$ and $h$ ). It is important to define DP. DP must along the direction of the design size or adjustable assembly dimensions. These dimensions will be an important part of the multi-dimensional vector loop and vector equation.

\section{Multi-dimensional vector loop and vector equation}

According to product's assembly sequence and locating, the vector direction and the position of DRF can be determined. A dimension vector loop is formed by connecting the deviation transfer paths of an assembly product. The vector loop can effectively describe the assembly deviation transfer path.

Dimension vector loop is divided into closed loop and open loop. Closed loop describes relations between nominal dimensions of parts and assembly dimensions. Open loop describes the influence of parts' nominal dimensions to the key characteristics in the assembly. Modeling rules for dimension vector loops include:

1) Loops must pass through every part and every joint in the assembly;

2) A single vector loop pass through the same joint no more than once, but it may start and end in the same part; 
3) If a vector loop includes the exact same dimension twice, in opposite directions, the dimension must be omitted;

Assuming that an assembly contains part dimensions $\left\{a_{1}, a_{2}, \ldots, a_{n}\right\}$, and assembly dimensions $\left\{U_{l}\right.$, $\left.U_{2}, \ldots, U_{m}\right\}$, multidimensional vector closed loops can be expressed as

$$
h_{D}=D\left(a_{1}, a_{2}, \mathrm{~L}, a_{n}, U_{1}, U_{2}, \mathrm{~L}, U_{m}\right)=0
$$

where $D$ is the vector direction of dimensions, for example, in three-dimensional space, $D=(u, v, w, \alpha$, $\beta, \gamma)$, in two-dimensional space, $D=(x, y, \theta)$ 。 The parameters $(u, v, w, x$ and $y)$ are location parameters of a vector. The parameters $(\alpha, \beta, \gamma$ and $\theta)$ are direction parameters of a vector.

Multidimensional vector open loops can be expressed as

$$
\text { Gap }=\Gamma\left(a_{1}, a_{2}, \mathrm{~L}, a_{n}, U_{1}, U_{2}, \mathrm{~L}, U_{m}\right)
$$

where $\Gamma$ is the vector direction along the open loop distance.

\section{Modeling example}

Taking an inner wing flap rocker as an example, the deviation transfer paths, multi-dimensional vector loop and vector equation are established.

The inner wing flap rocker mainly contains five components: $P_{\text {wing body }}, P_{\text {front connector }}, P_{\text {back connector }}, P$ rocker, and $P_{\text {pin }}$, shown in Fig. 3. The deviation sources are $a_{1}, a_{2}, \ldots, a_{13}$ and $\stackrel{\perp}{\perp} 0.2 \mathrm{~A}$.

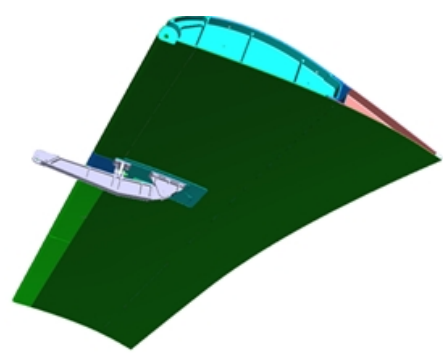

(a) an i nner wing $\mathrm{fl}$ ap rocker

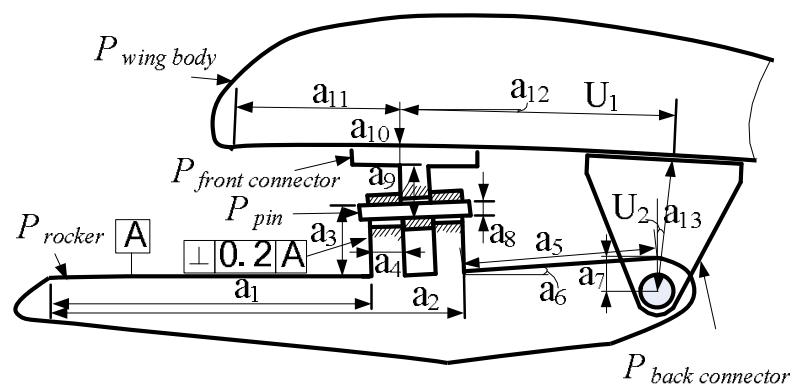

(b) A sect i onal vi ew

FIGURE 3: An inner wing flap rocker model

First of all, DPs are established: according to assembly constraints, the connection types and joints $\left(J\left(P_{\text {rocker }}, P_{\text {pin }}\right), J\left(P_{\text {pin }}, P_{\text {front connector }}\right), J\left(P_{\text {front connector }}, P_{\text {wing body }}\right), J\left(P_{\text {wing body }}, P_{\text {back connector }}\right)\right.$ and $J\left(P_{\text {back }}\right.$ connector,$\left.\left.P_{\text {rocker }}\right)\right)$ in the assembly are located; $D R F s\left(D R F_{\text {rocker }}, D R F_{\text {pin }}, D R F_{\text {front connector, }} D R F_{\text {wing body }}\right.$ and $D R F_{\text {back connector }}$ ) are defined based on factors such as component design references and assembly locating datum; $D P s$ from joints to $D R F$ s are created along the nominal dimension vector directions, as shown in Fig. 4.

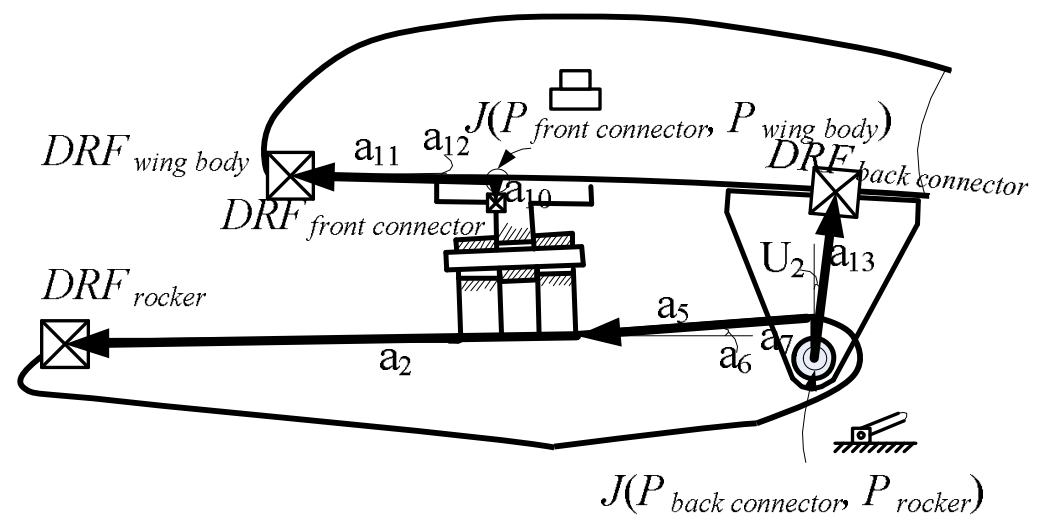

FIGURE 4: $D R F s$ and $D P S$ 
Then, the deviation transfer paths and multidimensional vector loops are created based on the DPs, as shown in Fig. 5. Fig. 5 (a) shows assembly deviation transfer vector closed loop. Because of $\perp 0.2 \mathrm{~A}, a_{14}$ become a deviation source. Figure 5 (b) shows assembly deviation transfer vector open loop. Gap means the distance from $D R F_{\text {wing body }}$ to $a_{l}$.

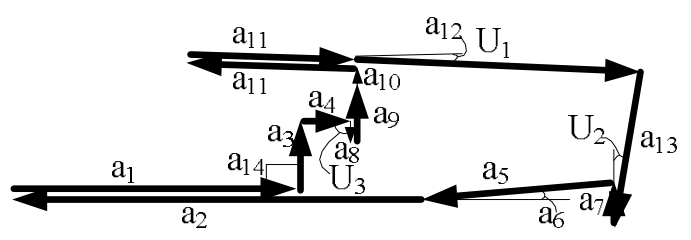

(a) The closed loop

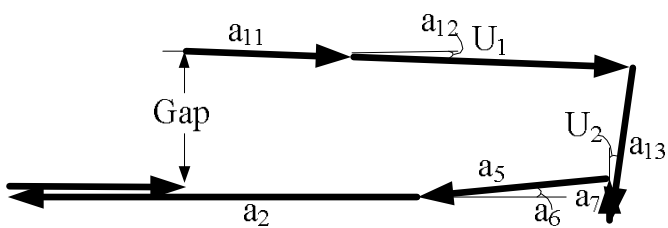

(b) The open loop

FIGURE 5: Deviation transmission vector loops

Finally, vector equations are generated as shown in (3).

$$
\begin{aligned}
& h_{x}=a_{1} \cos (0)+a_{3} \cos \left(a_{14}\right)+a_{4} \cos (0)+a_{8} \cos \left(-U_{3}\right)+a_{9} \cos (90)+a_{10} \cos (90)+ \\
& U_{1} \cos \left(-a_{12}\right)+a_{13} \cos \left(-U_{2}-90\right)+a_{7} \cos (90)+a_{5} \cos \left(-180+a_{6}\right)+a_{2} \cos (-180)=0 ; \\
& h_{y}=a_{1} \sin (0)+a_{3} \sin \left(a_{14}\right)+a_{4} \sin (0)+a_{8} \sin \left(-U_{3}\right)+a_{9} \sin (90)+a_{10} \sin (90)+ \\
& U_{1} \sin \left(-a_{12}\right)+a_{13} \sin \left(-U_{2}-90\right)+a_{7} \sin (90)+a_{5} \sin \left(-180+a_{6}\right)+a_{2} \sin (-180)=0 ; \\
& h_{\theta}=0+a_{14}-90-U_{3}+180-\left(90+a_{12}\right)-90-\left(180-U_{2}\right)+90-a_{6}+180=0 \\
& \text { Gap }=\left(a_{11}+U_{1}\right) \sin \left(-a_{12}\right)+a_{13} \sin \left(-U_{2}-90\right)+a_{7} \sin (90)+a_{5} \sin \left(-180+a_{6}\right) 。
\end{aligned}
$$

3 )

Assembly deviation source sensitivities can be solved by a first-order Taylor's series expansion and matrix operation of the vector equations, which is the base of the reliable assembly precision prediction. How to use this method, perform precision prediction and control, will be the direction of future research.

\section{Conclusions}

Introducing the concept of vector loop in the process of establishing the assembly deviation transfer path, effect of assembly constraints, the benchmark reference structure and assembly joint to assembly deviation transfer paths is given full consideration. The process of datum reference architecture definition, assembly joint definition, deviation transfer reference path construction, assembly vector loop and vector equation building is described in detail. The logic between part size tolerances, assembly positioning information and assembly precision is revealed.

The assembly deviation transfer path is described by the vector loop, providing a reliable model to support the follow-up assembly precision prediction and control in three-dimensional environment.

\section{References}

[1] YU DING, JIONGHUA JIN, DARIUSZ CEGLAREK, et al. Process-oriented tolerancing for multi-station assembly systems[J]. Iie Transactions, 2005, 37(6):493-508.

[2] Liu Y. HIERACHICAL REPRESENTATION MODEL AND ITS REALIZATION OF TOLERANCE BASED ON FEATURE[J]. Chinese Journal of Mechanical Engineering, 2003, 39.

[3] Anselmetti B. Generation of functional tolerancing based on positioning features[J]. Computer-Aided Design, 2006, 38(8):902-919.

[4] Liu W. Mechanism Analysis of Deviation Sourcing and Propagation for Mechanical Assembly[J]. Journal of Mechanical Engineering, 2012, 48(1):156-168. 
[5] Liu J, Jin J, Shi J. State Space Modeling for 3-D Variation Propagation in Rigid-Body Multistage Assembly Processes[J]. IEEE Transactions on Automation Science \& Engineering, 2010, 7(2):274-290.

[6] Mansuy M, Giordano M, Hernandez P. A new calculation method for the worst case tolerance analysis and synthesis in stack-type assemblies.[J]. Computer-Aided Design, 2011, 43(9):1118-1125. 